\title{
CARACTERIZAÇÃO DOS ATRIBUTOS QUÍMICOS DE UM HORIZONTE ANTRÓPICO EM GLEISSOLOS DO NORDESTE PARAENSE
}

\author{
Leane Castro de Souza ${ }^{1}$ Luma Castro de Souza ${ }^{2}$; Alexandre Roger de Araújo Galvão ${ }^{3}$; \\ Raimundo Leonardo Lima de Oliveira ${ }^{4}$; Antonia Vanderlane Albuquerque da Costa $^{5}$; \\ Antonio Robson Moreira ${ }^{6}$; Herdjania Veras de Lima ${ }^{7}$.
}

\footnotetext{
${ }^{1}$ Universidade Federal Rural da Amazônia. Capitão Poço, Pará, Brasil. leany_castro11@ @ hotmail.com

${ }^{2}$ Universidade Federal Rural da Amazônia. Belém, Pará, Brasil. lumasouza30@ hotmail.com

${ }^{3}$ Universidade Federal Rural da Amazônia. Capitão Poço, Pará, Brasil. alexandre.entomologia@ @otmail.com

${ }^{4}$ Universidade Federal Rural da Amazônia. Capitão Poço, Pará, Brasil. raimundoleonardoufra@ @otmail.com

${ }^{5}$ Universidade Federal Rural da Amazônia. Capitão Poço, Pará, Brasil. delanealbuquerque @ hotmail.com

${ }^{6}$ Universidade Federal Rural da Amazônia. Capitão Poço, Pará, Brasil. robson.n.13@ hotmail.com

${ }^{7}$ Universidade Federal Rural da Amazônia. Belém, Pará, Brasil. herdjania.veras@ufra.edu.br
}

\begin{abstract}
RESUMO: O objetivo do trabalho foi comparar atributos químicos dos solos de terra preta de índio com solos não antropogênicos. As coletas de solo foram realizadas no sitio arqueológico Jabuti e na área adjacente ao sítio em Bragança/PA. O solo desse sítio pertence à Ordem dos Gleissolos. As coletas foram realizadas em três áreas do sitio arqueológico (E1S1; E1S2 e área adjacente). Para esse estudo foram realizadas coletas a 5-10 $\mathrm{cm}$ de profundidade. Foram retiradas dez amostras simples para compor uma amostra composta. O delineamento utilizado foi inteiramente casualizado, sendo três áreas de estudo e um tipo de solo com 10 repetições, sendo que cada área de coleta dentro do sitio mede $10 \mathrm{~m}$. Foram calculadas as variáveis: soma de bases trocáveis, saturação por base, alumínio trocável, saturação por alumínio e CTC efetiva e CTC a pH 7,0. Os valores médios de soma de bases, saturação por bases foram maiores nas áreas de Terra Preta de Índio. Todavia, os valores médios de $\mathrm{m} \%$ foram menores nos solos antropogênicos. Enquanto, os valores médios de $\mathrm{Al}^{3+}$ foram menores no ponto E1S1 do sitio arqueológico. A CTC efetiva foi maior nas áreas E1S1e E2S1 (29,21 e 20,93 $\mathrm{cmol}_{\mathrm{c}} / \mathrm{dm}^{3}$ respectivamente) e menor na área $\left(5,55 \mathrm{cmol}_{\mathrm{c}} / \mathrm{dm}^{3}\right)$. Enquanto os valores médios de CTC a $\mathrm{pH} 7$ foi maior nas áreas E1S1e E2S1 (41,88; 32,39 $\mathrm{cmol}_{\mathrm{c}} / \mathrm{dm}^{3}$ respectivamente) e menor na área Adjacente (15 $\left.\mathrm{cmol}_{\mathrm{c}} / \mathrm{dm}^{3}\right)$. As terras pretas de índio apresentam são mais férteis do que os solos não antropogênicos.
\end{abstract}

PALAVRAS-CHAVE: atributos químicos, fertilidade, sítio arqueológico.

\section{CHARACTERIZATION OF CHEMICAL ATTRIBUTES OF A HORIZON ANTHROPIC IN NORTHEAST GLEYSOLS PARAENSE}

\begin{abstract}
The aim of this study was to compare the chemical properties of soils of Indian black earth soils with no anthropogenic. The soil samples were taken in Tortoise archaeological site in Bragança / PA, which has a total area of $100 \mathrm{~m} \times 100 \mathrm{~m}$. The soil of this website belongs to the Order of Gleissolos. Samples were collected in three areas of the archaeological site (E1S1; E1S2 and adjacent area). For this study were collected at $5-10 \mathrm{~cm}$ depth. Ten simple samples were taken to compose a composite sample. The experimental design was completely randomized, with three areas of study and a soil type with 10 repetitions, each collection area within the site measures $10 \mathrm{~m}$. Total exchangeable bases, base saturation, exchangeable aluminum, aluminum saturation and effective CEC and CEC at $\mathrm{pH}$ 7: the variables were calculated. The average values of sum of bases, base saturation were higher in areas of Dark Earth. However, the average values of $\mathrm{m} \%$ were lower in
\end{abstract}


anthropogenic soils. While the average values of $\mathrm{Al}^{3+}$ were lower in point E1S1 the archaeological site. The effective CEC was higher in areas E1S1 and E2S1 (29.21 and 20.93 $\mathrm{cmolc} / \mathrm{dm}^{3}$ respectively) and lowest in the area $\left(5.55 \mathrm{cmolc} / \mathrm{dm}^{3}\right)$. While the average values of CEC at pH 7 was higher in areas E1S1 and E2S1 (41,88 and 32,39 $\mathrm{cmolc} / \mathrm{dm}^{3}$ respectively) and Adjacent the lower $\left(15 \mathrm{cmolc} / \mathrm{dm}^{3}\right)$ area. The black earth indiums present are more fertile than non-anthropogenic soils.

KEYWORDS: archaeological site, chemical characteristics, fertility.

\section{INTRODUÇÃO}

A maioria dos solos agricultáveis da Amazônica apresenta alta acidez, baixa capacidade de troca de cátions (CTC), consequentemente, baixa fertilidade e potencial produtivo. O grau de fertilidade do solo é um fator que limita a produtividade e sustentabilidade ambiental e econômica (MADARI et al., 2009). Todavia, são encontrados nessa região solos que apresentam alta fertilidade e boa retenção de nutrientes, o que os torna uma alternativa viável na busca de uma agricultura sustentável, tais solos são denominados de Terra Preta de Índio (TPI), Terra Preta Arqueológica (TPA) ou Terra Preta (TP) (KERN; KÄMPF, 1989).

Esses solos possuem como característica marcante a elevada fertilidade natural, coloração escura e fragmentos de cerâmica e/ou líticos inseridos nos horizontes mais superficiais do solo (KAMPF; KERN, 2005).

A elevada fertilidade e principalmente a sustentabilidade das áreas de TPI é atribuída ao elevado nível de matéria orgânica e as suas propriedades físico- químicas como, a elevada reatividade das frações húmicas (CUNHA et al., 2009). A fertilidade desses solos tem levado os pequenos produtores da região amazônica cultivar culturas de subsistências, tais como o milho (Zea mays L.), o feijão (Phaseolus vulgaris L.) e a mandioca (Manihot esculenta Crantz) (COSTA, 2011).

Do ponto de vista cultural esses solos são importantes registros do processo de habitação na região amazônica, e essas podem tornar mais claros questionamentos a respeito da distribuição da população, capacidade de suporte do solo, padrões de assentamentos e o uso da terra por populações pré-colombianas (LIMA et al., 2002).

O objetivo do trabalho foi comparar atributos químicos dos solos de terra preta de índio com solos não antropogênicos.

\section{MATERIAL E MÉTODOS}

As amostras de solo da Terra Preta de Índio foram coletadas no sitio Arqueológico Jabuti na Reserva Extrativista Marinha Caeté-Taperuçu, 
registrado em 2008, e as amostras correspondentes à área considerada como controle foram coletadas ao lodo do Sítio, por isso foi chamada de área adjacente. $\mathrm{O}$ solo do Sítio Arqueológico Jabuti pertence à Ordem dos Gleissolos, apresentando uma área total de $100 \mathrm{~m} \times 100 \mathrm{~m}$. A área adjacente ao sitio pertence à mesma classe de solo. O sitio fica localizado em uma ilha de terra firme no litoral Bragantino a 240km de Belém.

O sítio apresenta as seguintes coordenadas geográficas: $46^{\circ} 40^{\prime} 19.8^{\prime \prime} \mathrm{W} /$ $0^{\circ} 55^{\prime} 39.5 " \mathrm{~S}$, sendo que a margem de erro é de 5m, South America Datum 69. As coletas foram realizadas em três áreas do sitio arqueológico, essas foram chamadas de E1S1, E1S2 e área adjacente.

As amostras foram coletadas no sentido Leste-Oeste em relação às áreas de coleta. A área adjacente está situada sob uma floresta secundaria, a poucos metros da faixa de ocorrência da TPI. Em décadas passadas, houve a retirada da vegetação primária para formação de pastagens destinadas à pecuária.

Atualmente na área do sítio Jabuti, crescem capoeira e florestas secundárias com grande quantidade de palmeiras tais como inajá (Maximiliana martiana $\mathrm{H}$. Karst.) e babaçu (Orbignya oleifera Burret). Ainda nos dias atuais podemos encontrar a floresta original em pequenos tratos isolados, sendo que as mesmas correspondem ao tipo geral das Florestas Tropicais Úmidas, subtipo Floresta Densa (SILVEIRA et al., 2011).

Para esse estudo foram realizadas coletas a 5-10 $\mathrm{cm}$ de profundidade. Por isso foram feito um transecto de $10 \mathrm{~m}$ no sentido horizontal as trincheiras das áreas de estudo. As coletas foram feitos a cada 1 m em cada área, totalizando 10 amostras de estrutura deformada. Foram retiradas 10 amostras simples para compor uma amostra composta, para realização das análises.

O delineamento utilizado foi inteiramente casualizado, sendo três áreas de estudo e um tipo de solo com 10 repetições, sendo que cada área de coleta dentro do sitio mede $10 \mathrm{~m}$.

As análises químicas foram realizadas em triplicata. Foram calculadas as variáveis: soma de bases trocáveis (SB); saturação por base (V\%); alumínio trocável $\left(\mathrm{Al}^{+3}\right)$ e saturação por alumínio (m\%) (EMBRAPA, 1997). As médias foram submetidas a analise de variância (teste F) no nível de significância de $5 \%$ de probabilidade.

\section{RESULTADOS E DISCUSSÃO}

Observa-se na Figura 1A, que os valores médios de saturação por bases nas áreas E1S1, E2S1 e Adj. foram de 28,45; 17,03 e $3,30 \mathrm{cmol}_{\mathrm{c}} / \mathrm{dm}^{3}$ respectivamente. Os valores médios de saturação por bases 
(V\%) nas áreas E1S1, E2S1 e Adj. foram de 67,$91 ; 52,58$ e $21,99 \mathrm{cmol}_{\mathrm{c}} / \mathrm{dm}^{3}$ respectivamente (Figura 1B).

Os valores médios de saturação por alumínio nas áreas E1S1, E2S1 e Adj. foram de 32,09; 47,42 e 78,01 $\mathrm{cmol}_{\mathrm{d}} / \mathrm{dm}^{3}$ respectivamente (Figura 1D). Sendo que as áreas E1S1 e E2S1 apresentaram maior soma de bases (SB) e saturação por bases (V\%) quando comparadas a área adjacente. No entanto, as áreas E1S1 e E2S1 apresentam menores valores de saturação por alumínio quando comparado à área adjacente. Campos et al. (2012) também observaram maiores valores de soma de bases nos horizontes antropogênicos. Para o mesmo autor a percentagem de saturação de bases (V\%) apresentaram maiores valores nos horizontes antrópicos dos sítios estudados, quando comparado aos solos não antropogênicos.

Os valores médios de $\mathrm{Al}^{3+}$ trocável foram maiores em um dos pontos de coleta da TPI (E2S1- 3,9 $\left.\mathrm{cmol}_{\mathrm{c}} / \mathrm{dm}^{3}\right)$ e no ponto de coleta do solo adjacente (2,55 $\mathrm{cmol}_{\mathrm{c}} / \mathrm{dm}^{3}$ ). Enquanto no outro ponto de coleta da TPI (E1S1 - 0,77 $\left.\mathrm{cmol}_{\mathrm{C}} / \mathrm{dm}^{3}\right)$ esse valor foi menor (Figura 1C). Campos et al. (2012) observaram que os teores de $\mathrm{Al}^{3+}$ trocável foram menores em todos os horizontes das terras pretas de índio quando comparados às áreas de solos adjacentes. Esse fato ocorre devido a acumulação de matéria orgânica no solo causar a redução dos teores de alumínio trocável na solução do solo (MIYAZAWA et al., 1993), por meio do processo de complexação do íon $\mathrm{Al}^{3+}$ pela matéria orgânica.

Enquanto os valores médios de saturação por alumínio $(\mathrm{m} \%)$ na área adjacente foi maior $(77,79 \%)$ do que os encontrados na TPI (E2S1- 47,18 e E1S132,07\%) (Figura 1C). Esses resultados mostram que, em geral, as TPIs não apresentam problemas com toxidez de alumínio (FALCÃO et al., 2009).

Os valores médios de CTC efetiva nas áreas E1S1, E2S1 e Adjacente foram de 29,$21 ; 20,93$ e $5,55 \quad \mathrm{cmol}_{\mathrm{c}} / \mathrm{dm}^{3}$ respectivamente (Figura 2A). Enquanto os valores médios de CTC a pH 7 nas áreas E1S1, E2S1 e Adjacente foram de 41,88; 32,39 e $15 \mathrm{cmol}_{\mathrm{c}} / \mathrm{dm}^{3}$ respectivamente (Figura 2B). Sendo que as áreas E1S1 e E2S1 apresentaram maior soma de bases (SB), CTC efetiva (t) e CTC a pH $7(t)$ quando comparadas a área adjacente.

Campos et al. (2012) também observaram maiores valores de soma de bases foram nos horizontes antropogênicos. Para o mesmo autor a percentagem de saturação de bases (V\%) apresentaram maiores valores nos horizontes antrópicos dos sítios estudados, quando comparado aos solos não antropogênicos.

Revista Agroecossistemas, v. 5, n. 2, p. 1-7, 2013 

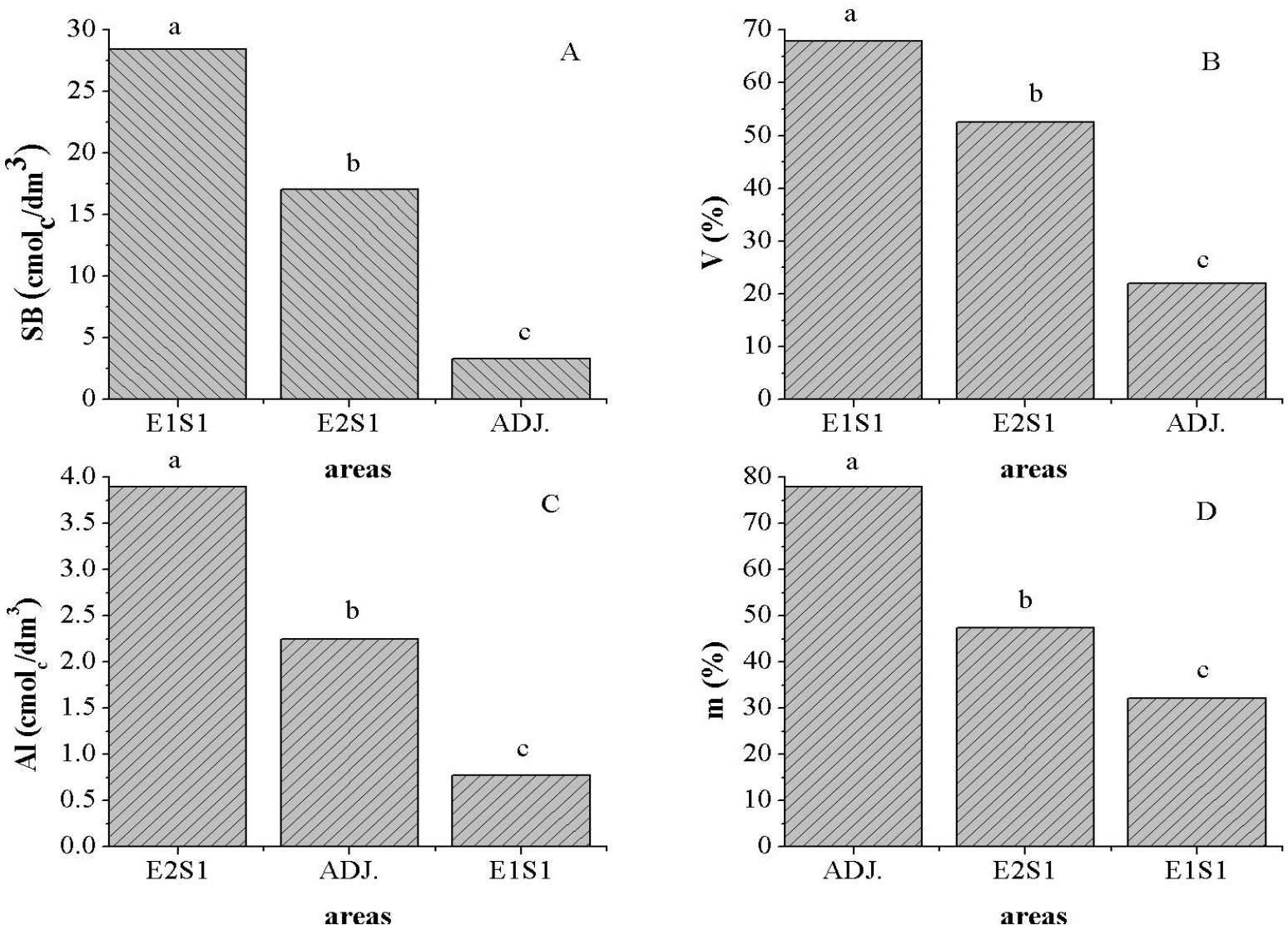

Figura 1. Valores médios de soma de bases (SB) (A), Saturação por base (v\%) (B), alumínio trocável $\left(\mathrm{Al}^{3+}\right)(\mathrm{C})$ e saturação por alumínio (D) em solos de Terra Preta de Índio no Pará. Letras minúsculas iguais não diferem estatisticamente ao nível de $5 \%$ de probabilidade, através do teste de Tukey.

Os maiores valores da CTC não se deve apenas ao elevado teor de material orgânico presente nesses solos, mas também de uma maior densidade de cargas por unidade de carbono (LIANG et al., 2006) encontrado nesses solos. Esta propriedade do carbono orgânico é característica de solos que apresentam alto conteúdo de carvão pirogênico como a Terra Preta de Índio (CUNHA et al., 2007). Para Lehmann et al. (2005) esta elevada densidade de carga pode, no começo, provocar uma maior oxidação do próprio carbono pirogênico ou por meio de adsorção do carbono não pirogênico. Esses processos vêm sendo observado nos solos de Terras Pretas de Índio (LIANG et al., 2006). Campos et al. (2012) observaram que a CTC a pH 7,0 nas áreas de terras pretas arqueológicas apresentaram maiores valores quando comparados aos ambientes não antropogênicos. 

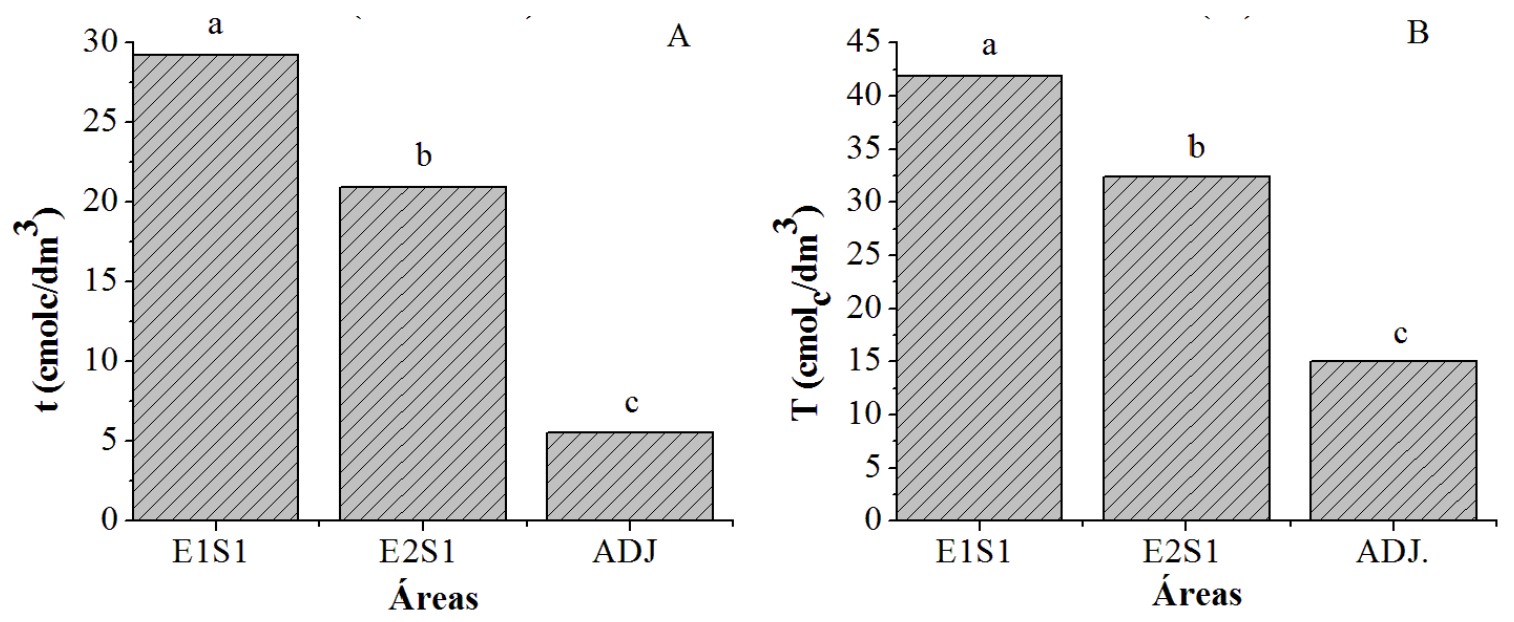

Figura 2. Valores médios de CTC efetiva (t) (A) e CTC a pH 7 (T) (B) em solos de Terra Preta de Índio no Pará. Letras minúsculas iguais não diferem estatisticamente ao nível de 5\% de probabilidade, através do teste de Tukey.

\section{CONCLUSÃO}

As terras pretas arqueológicas apresentam maiores valores de soma de bases, saturação por bases e menores valores de alumínio trocável, saturação por alumínio e CTC efetiva e CTC a pH 7 em relação aos solos não antropogênicos, conferindo-lhes maior fertilidade.

\section{REFERÊNCIAS}

CAMPOS, M. C. C.; SANTOS, L. A. C.; SILVA, D. M. P.; MANTOVANELLI, B. C.; SOARES, M. D. R. Caracterização física e química de terras pretas arqueológicas e de solos não antropogênicos na região de Manicoré, Amazonas. Revista Agro@mbiente Online., v. 6, n. 2, p. 102-109, 2012. Disponível em: http://revista.ufrr.br/index. php/agroambiente/article/view/682/776. Acesso em: 12 mar. 2014.

COSTA, A. R. Formas de Fósforo do Solo em Sítios de Terra Preta
Arqueológica na Amazônia Oriental, Belém, 2011. 116f. Dissertação (Mestrado em Agronomia) - Universidade Federal Rural da Amazônia, Belém, 2011.

CUNHA, T. J. F.; MADARI, B. E.; CANELLAS, L. P.; RIBEIRO, L. P.; BENITES, V.M.; SANTOS, G. A.; Soil organic matter and fertility of anthropogenic dark earths (terra preta de indio) in the Brazilian Amazon basin. Revista Brasileira de Ciência do Solo. v. 33, n. 1, p.85-93, Feb. 2009. Também disponível em: http://www.scielo.br/scielo. php?script=sci_arttext\&pid=S0100068320 09000100009.

CUNHA, T. J. F.; NOVOTNY, E. H.; MADARI, B. E.; BENITES, V. de M.; MARTIN NETO, L.; SANTOS, G. de A. $\mathrm{O}$ carbono pirogênico. In: TEIXEIRA, W. G.; KERN, D. C.; MADARI, B. E.; LIMA, H. N.; WOODS, W. (Ed.). As terras pretas de índio da Amazônia: sua caracterização e uso deste conhecimento na criação de novas áreas. Manaus: Embrapa Amazônia Ocidental, 2007. p. 263-284.

EMBRAPA. Serviço Nacional de Levantamento e Conservação de Solos. 
Manual de métodos de análise de solo. Rio de Janeiro: EMBRAPA-SNLCS, 1997, $212 p$.

FALCÃO, N.; MOREIRA, A.; COMENFORD, N. B. A Fertilidade dos Solos de Terra Preta de Índio da Amazônia Central. In: TEIXEIRA, W.G.; KERN, D.C.; MADARI, B.E.; LIMA, H.N.; WOODS, W. (Eds.). As Terras Pretas de Índio da Amazônia: sua caracterização e uso deste conhecimento na criação de novas áreas. Manaus: EDUA: Embrapa Amazônia Ocidental, 2009, v. 1, p. 172188.

KÄMPF, N.; KERN, D. C. O. Solo como registro da ocupação humana pré histórica na Amazônia. In: TORRADO, P. V.; ALLEONI, L. R. F; COOPER, M.; SILVA, A. P.; CARDOSO, E. J. (Org). Tópicos em Ciência do solo. Viçosa: Sociedade Brasileira de Ciência do Solo, v. 6, p. 277-320, 2005.

KERN, D.C.; KÄMPF, N. O efeito de antigos assentamentos indígenas na formação de solos com terra preta arqueológicas na região de Oriximiná PA. Revista Brasileira de Ciência do Solo, v. 13, p. 219-225, 1989.

LEHMANN, J.; LIANG, B.Q.; SOLOMON, D.; LEROTIC, M.; LUIZAO, F.; KINYANGI, J.; SCHAFER, T.; WIRICK, S.; JACOBSEN, C. Near-edge xray absorption fine structure (NEXAFS) spectroscopy for mapping nano-scale distribution of organic carbon forms in soil: Application to black carbon particles. Global Biogeochemical Cycles, Washington, DC, v. 19, p. 12, 2005.

LIANG, B.; LEHMANN, J.; SOLOMON, D.; KINYANGI, J.; GROSSMAN， J.; O`NEILL, B.; SKJEMSTAD, J.O.; THIES, J.; LUIZÃO, F.J.; PETERSEN, J.; NEVES, E.G. Black carbon increases cations exchange capacity in soils. Soil
Science Society of America Journal, v. 70, p. 1719-1730, 2006.

LIMA， H.N.; SCHAEFER， C.E.R.; MELLO, J.W.V.; GILKES, R.J.; KER, J.C. Pedogenesis and Pre-Colombian land use of "Terra Preta Anthrosols" ("Indian black earth") of Western Amazonia. Geoderma, Amsterdam, v. 110, p. 1-17, 2002.

MANDARI, B. E.; SOMBROEK, W.; WOODS, W.I. Research on anthropogenic dark earths soils. Cold be a solution for sustainable agricultural development in the Amazon? In: GLASER, B.; WOODS, W. I. Amazonian dark earth: exploration in space and time. Berlin: Springer, 2004, p.169-180.

MIYAZAWA, M.; PAVAN, M.A.; CALEGARI, A. Efeito do material vegetal na acidez do solo. Revista Brasileira de Ciência do Solo, v. 17, p.411-416, 1993.

SILVEIRA, M. I.; OLIVEIRA, E. R.; KERN, D. C.; COSTA, M. L.; RODRIGUES, S. F. S. O sítio Jabuti, em Bragança, Pará, no cenário arqueológico do litoral amazônico. Boletim do Museu Paraense Emílio Goeldi. Ciências Humanas, v. 6, n. 2, p. 335-345, 2011. 